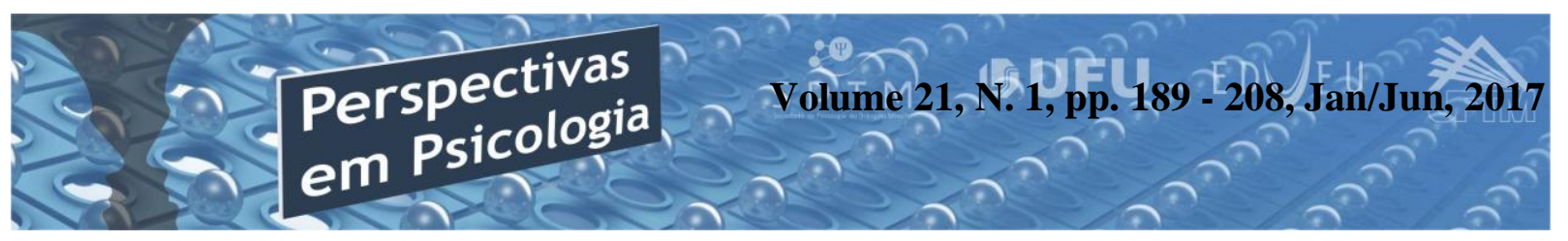

\title{
A PRÁTICA DO CUIDADO: A PSICOLOGIA PELO OLHAR DO PSICÓLOGO E DE EQUIPES DE SAÚDE
}

\author{
Marina Kohlsdorf \\ Rayanne Pollyane Chagas Borges \\ (Centro Universitário UniCeub - Brasília)
}

\begin{abstract}
Resumo
Este estudo objetiva investigar percepções do psicólogo sobre sua prática em equipes multidisciplinares e a observação destas equipes sobre a psicologia. Trata-se de pesquisa quantitativa, que utilizou questionários respondidos por 29 psicólogos e 36 profissionais da saúde. Os resultados demonstraram que o psicólogo é considerado importante para a composição da equipe, mas sua atuação não foi claramente definida. $\mathrm{O}$ trabalho em equipe é fundamental para o reconhecimento das diferentes áreas de atuação, porém muitas vezes a equipe desconhece funções desempenhadas pelo psicólogo. Destaca-se que a prática psicológica em saúde precisa ser ampliada, incluindo maior presença destes profissionais em diferentes equipes.
\end{abstract}

Palavras chave: psicologia da saúde; interdisciplinaridade; ambiente hospitalar.

\section{Abstract \\ The caring practice: Psychology according to psychologists and health care team}

This paper investigates psychologist's perception upon psychological practice in multidisciplinary health care teams, and these teams observation related to Psychology. This is a quantitative research, which used questionnaires answered by 29 psychologists and 36 health care professionals. Results show that the psychologist is considered relevant in health care team composition, however the psychologist functions could not be clearly defined. The health care team work is essential for recognizing different areas of occupation, however usually the health care team does not know the functions developed by the psychologist. It is highlighted that health psychology practice must be expanded, including the increase of the presence of psychologists in different health care teams.

Keywords: health psychology; interdisciplinarity; hospital environment.

\section{Introdução}

A Psicologia é uma ciência que pode contribuir para trabalhar a subjetividade das relações que circundam a humanização da assistência, uma vez que os estudos desta área visam uma maior compreensão das relações humanas e oferecem intervenções que facilitam o processo de comunicação entre os 
profissionais da saúde e o paciente (Feijó, 2006). Para a atuação eficaz da psicologia no contexto de promoção de saúde e prevenção de doenças, é imprescindível que o psicólogo conheça as atribuições da equipe de profissionais e que a equipe conheça as atribuições do psicólogo.

A dinâmica da relação entre pacientes e profissionais da saúde fundamenta-se na herança de um modelo que atribui ao adoecer a influência apenas dos aspectos neurofisiológicos e bioquímicos do sistema nervoso. Este modelo, chamado biomédico e tecnicista, embasou a medicina desde o século XVII, apresentando uma compreensão do sofrimento humano a partir de sua estrutura orgânica. Logo, a atitude do médico frente à pessoa enferma priorizava a indicação de tratamento farmacológico, sem considerar a interferência dos fatores que envolvem as vivências históricas do paciente (Feijó, 2006). De acordo com este modelo biomédico, as doenças advêm do exterior do corpo, invadindo-o e causando mudanças físicas dentro do mesmo, tendo também origem em mudanças físicas internas involuntárias. O tratamento das doenças é caracterizado como responsabilidade médica e são exemplos de tratamento a vacinação, cirurgias, quimioterapias, entre outros, todos com a finalidade de mudar o estado físico do doente (Feijó, 2006).

Segundo o modelo biomédico, não existe uma continuidade entre a doença e a saúde, ambas são distintas. Este considera que a mente e o corpo funcionam independentemente, ou seja, a mente não é capaz de influenciar o estado físico, sendo encarada como algo abstrato e relacionada com os sentimentos e pensamentos. Por sua vez, o corpo é visto em termos de substâncias físicas, como pele, músculos, ossos, cérebro e órgãos. Assim, a doença pode ter consequiências psicológicas, mas não etiologias psicológicas. Por exemplo, o câncer pode causar infelicidade, contudo o estado emocional do paciente não é visto como algo que está relacionado nem com a origem da doença e nem com a sua progressão (De Marco, 2010).

Czeresnia (2003) ressalta que o modelo biopsicossocial de compreensão do fenômeno saúde-doença, que se desenhou como tentativa de superar o modelo biomédico, pretendeu oferecer a possibilidade de uma compreensão mais integrada das representações e da experiência de saúde-doença, refutando os pensamentos dualistas e reducionistas. Esta perspectiva que tem como referência o 
modelo biopsicossocial tem-se afirmado progressivamente. A emergência de novas proposições sobre o processo saúdedoença, como por exemplo, a psicologia da saúde, veio desafiar a cisão mente-corpo ao propor um papel para a mente, tanto na causa como no tratamento da doença, proporcionando uma visão integral do ser humano. Este modelo representou uma tentativa para integrar o lado psicológico ("psico") e o meio ambiente ("social") ao modelo biofisiológico ("bio"). (Castro \& Bornholdt, 2004; De Marco, 2010).

$\mathrm{O}$ adoecer, portanto, implica o rompimento das defesas pessoais, ameaçando o equilíbrio psicológico do paciente ao provocar um choque emocional diante, por exemplo, do abandono das atividades cotidianas, do afastamento das suas relações sociais, da sua permanência no leito, da falta de informação diante de sua enfermidade, das falhas na comunicação entre equipe e paciente, da sua dependência perante os cuidados e proteção dos familiares e profissionais (Feijó, 2006).

Segundo Tonetto e Gomes (2007), o hospital é uma instituição complexa, que envolve um grande número de especialidades. No contexto de saúde, o psicólogo deve buscar estabelecer um contato mais próximo com outras profissões. A saúde não é de competência de um único profissional, ela é uma prática interdisciplinar e os profissionais das muitas e diferentes áreas de atuação devem agregar-se em equipes de saúde (Saar \& Trevizan, 2007). A Psicologia, ao ser comparada com as demais especialidades que compõem uma equipe de saúde, é considerada uma ciência recente, o que pode ser uma explicativa para os psicólogos ainda possuírem pouca penetração em outras áreas, fazendo com que suas ações sejam pouco conhecidas neste meio (Castro \& Bornholdt, 2004; More \& cols., 2004; Saar \& Trevizan, 2007).

Conjuntamente com o enfoque da humanização do atendimento em saúde, a interdisciplinaridade é uma das bases da tarefa do psicólogo que adentra este contexto, partindo do pressuposto de que o indivíduo deve ser considerado biopsicossocial. Portanto, estas ações deveriam envolver profissionais de diferentes áreas em uma rede de complementaridade, onde são mantidas as exigências organizacionais unitárias.

Segundo More e cols (2004) o convívio dos diferentes integrantes das equipes de saúde multiprofissionais traz à tona os mais variados questionamentos com relação à postura destes profissionais, 
no que diz respeito a parâmetros de ação em comum, e que se traduzem em ações de saúde fragmentadas ou ambíguas, gerando situações de real desconfirmação das necessidades da população que procura atendimento no contexto públicocomunitário. Esta realidade tem gerado a necessidade de capacitação dos profissionais, no sentido de desenvolver e trabalhar diálogos multidisciplinares em direção aos interdisciplinares. O desafio é pensar na interdisciplinaridade, ou seja, na busca de um consenso das ações e o discurso das equipes, visando o desenvolvimento da comunicação entre os diferentes saberes entre os distintos profissionais.

Destaca-se neste contexto a necessidade de reconhecimento e valorização do saber e da intervenção de todos os membros da equipe e não simplesmente uma aceitação do discurso do outro em detrimento do seu, somando os pensamentos divergentes (More \& cols., 2004). Tais dificuldades têm gerado discussões sobre qual o modo mais apropriado da Psicologia se inserir nas equipes multidisciplinares (Romano, 1999). Para o autor uma primeira condição para o trabalho interdisciplinar efetivo do psicólogo é a clareza de suas atribuições e das expectativas concernentes a sua especificidade. No caso de estarem esclarecidas as atribuições do psicólogo, espera-se que ele seja capaz de se mostrar competente o suficiente para que sua prática seja vista como necessária (Chiattone, 2000; More \& cols., 2004 citados por Tonetto \& Gomes, 2007).

Para os psicólogos, pela sua relativa recente inserção no setor saúde, ainda não há uma definição clara do seu papel em cada um dos níveis de atenção, o que resulta em desconhecimento das possibilidades de atuação. Essencialmente, o desempenho na atenção básica se caracteriza pelo desenvolvimento de um trabalho da equipe de saúde na e com a comunidade através do modelo da vigilância da saúde, focando, sobretudo, ações de promoção à saúde e trabalhando também com prevenção e atenção curativa (Böing \& Crepaldi, 2010; Castro \& Bornholdt, 2004).

O psicólogo deparou-se com a necessidade de desenvolver habilidades que permitam o trabalho em equipe, visto que sua prática efetiva ocorria de forma predominantemente individualizada. Nessa nova realidade de trabalho, o psicólogo e os demais componentes da equipe experimentaram no seu cotidiano 
limitações profissionais e entenderam que os colegas de outras formações poderiam fornecer respaldo fundamental para a melhor compreensão do processo de intervenção neste contexto (Tonetto \& Gomes, 2007). Nessa mesma direção, Böing e Crepaldi (2010) apontam que os profissionais, à medida que se tornaram conscientes de que possuíam limites técnicos ou pessoais para enfrentar certos obstáculos, passaram a solicitar a ajuda da equipe de trabalho, o que transformou a resolução do problema e o enfrentamento da imprevisibilidade do processo saúdedoença em uma tarefa de equipe.

De uma forma geral, a equipe multidisciplinar pode ser definida como um grupo de profissionais que atuam de forma independente em um mesmo ambiente de trabalho, utilizando-se de comunicações informais, enquanto a atuação interdisciplinar pressupõe a integração dos diferentes saberes, superando a fragmentação da ciência tradicional, a partir da articulação das diferenças e reconhecimento da atuação das diferentes especificidades que trabalham em conjunto (More \& cols., 2004). Assim, uma excelente configuração de equipe pode estruturar-se quando seus membros se juntam em torno da humanização do atendimento a pacientes doentes e hospitalizados (AngeramiCamom \& cols., 2009).

De acordo com Fossi e Guareschi (2004), o trabalho em equipe mostra-se fundamental para o atendimento sistêmico, na medida em que médicos, enfermeiros, psicólogos, nutricionistas, assistentes sociais e os demais profissionais envolvidos estabelecem uma integração, para que a pessoa seja tomada como um todo, e possa ter um atendimento humanizado, contemplando assim, suas necessidades. A contribuição da psicologia da saúde não se limita ao usuário ou à instituição, mas às especificidades que auxiliam todo o trabalho da equipe (Castro \& Bornholdt, 2004).

A equipe é composta por diversos profissionais, incluindo aqueles que não assistem as pessoas diretamente, tais como equipe de higienização, radiologista, anestesista, entre outros. No entanto, consideraremos aqui a equipe multidisciplinar formada pelos profissionais que assistem diretamente os indivíduos: médicos, enfermeiros, psicólogos, nutricionista, assistente social, fisioterapeuta. Cabe salientar que a equipe multidisciplinar tem sua formação centrada nas necessidades da pessoa, portanto, ela não é pré-organizada. A demanda do enfermo é que fará com que os 
profissionais da saúde se integrem, com o propósito de satisfazer as necessidades globais do usuário, proporcionando seu bem-estar (Fossi \& Guareschi, 2004).

É a trajetória saúde-doença do indivíduo que definirá o enfoque de seu atendimento psicológico, que poderá ser preventivo, em promoção de saúde, pré ou pós-operatório, ambulatorial ou de enfermaria. É através desta consideração que o trabalho do psicólogo será delineado e implementado, considerando as necessidades individuais da pessoa (Angerami-Camon, 1987, citado por Fossi \& Guareschi, 2004).

O psicólogo integra a visão biopsicossocial do paciente que precisa de uma assistência integrada. Cada vez mais, a equipe de saúde implica importância aos fatores psicológicos, emocionais, sociais e culturais do paciente. Dessa forma, a presença do profissional de psicologia na equipe multidisciplinar já ocorre em muitas instituições de saúde (Castro \& Bornholdt, 2004; Rêgo, 2008). Entretanto, conforme salientado no trabalho de Saar e Trevizan (2007), as funções de médicos, enfermeiros e farmacêuticos são bastante conhecidas pelas equipes de saúde, porém o trabalho do psicólogo e suas funções ainda são pouco conhecidos pelos outros profissionais.

Para Angerami-Camon e cols. (2009), cabe ao psicólogo a responsabilidade da conquista do contexto de saúde como campo de sua atuação profissional. E esta perspectiva apenas se tornará real quando sua reflexão o levar ao encontro de parâmetros que o conduzam a uma atuação permeada pela multidisciplinaridade institucional. Romano (1999 citado por Tonetto \& Gomes, 2007), avaliando os dez anos de inserção do psicólogo na rede de saúde, afirmou que a instituição continua com interesse no psicólogo, mas ainda não sabe as funções específicas desta área de conhecimento. Segundo o autor há queixas entre psicólogos de que muitas das suas observações clínicas não são prontamente aceitas pelas equipes.

Com o redirecionamento das políticas de saúde no País, os profissionais psicólogos passam também a fazer parte desse repertório de recursos em saúde chamado SUS. Os profissionais psicólogos - tendo como responsabilidade a promoção do respeito à dignidade e à integridade do ser humano - são reconhecidos junto ao Conselho Nacional de Saúde (CNS) como profissionais de saúde de nível superior 
(Brasil, 1997 citado por Silva, 2008). Conforme Böing e Crepaldi, (2010), através do princípio da integralidade, portanto, o SUS abre portas para novos atores nas equipes de saúde. Para cuidar da saúde de forma integral, torna-se imprescindível que, no primeiro nível de atenção, haja equipes interdisciplinares que desenvolvam ações intersetoriais. $\mathrm{O}$ psicólogo nesse contexto oferece uma importante contribuição na compreensão contextualizada e integral do indivíduo, das famílias e da comunidade (Castro \& Bornholdt, 2004).

Segundo os dados do IBGE apresentados por Dimenstein (1998), o número de psicólogos participantes das equipes de saúde de nível superior de todos os estabelecimentos públicos e privados no ano de 1976 era de 726 em todo o Brasil, o que correspondia a $0,52 \%$ em relação ao total de categorias pesquisadas, valor inferior ao de outras categorias profissionais ditas "de apoio" ou paramédicas. Dimenstein (1998) cita que em 1988, foi aprovado na nova Constituição o SUS (Sistema Único de Saúde), que é a forma atualmente proposta de produzir serviços de saúde para o setor público de forma descentralizada, numa rede regionalizada e hierarquizada, priorizando-se o atendimento integral e as atividades preventivas. Nesse contexto, novas demandas de responsabilidade social estão sendo feitas aos psicólogos, o que nos leva ao questionamento de seus saberes, dos seus referenciais teóricos e dos seus modelos assistenciais e sua adequação à realidade do SUS. Pode-se considerar que à elementos contributivos a uma passagem da situação atual de uma ainda incipiente inserção do psicólogo no campo da saúde pública, para uma outra participação, mais coerente com o disposto nas diretrizes de formação, que prevê sua atuação no SUS, como membro integrante da equipe de saúde.

No Brasil, há relatos de inserção do psicólogo em hospitais desde a década de 1950, com um enfoque estritamente clínico (Castro \& Bornholdt, 2004). Aos poucos, o campo de atividades profissionais ficou mais vasto e permitiu que o serviço psicológico prestado levasse em conta a saúde física e psicológica da sociedade, ampliando as possibilidades de atuação para um âmbito educativo, preventivo e promotor de qualidade de vida, compreendendo assim a atuação do profissional dentro da área de psicologia da saúde e promovendo a visão de psicologia no contexto de políticas públicas e saúde mental (Baptista, 2003 citado por Rêgo, 2008; Castro \& Bornholdt, 2004). 
Em 1979, foi criada a divisão 38 da American Psychological Association, referente à Psicologia da Saúde, cujos objetivos básicos são avançar no estudo da Psicologia como disciplina que compreende a saúde e a doença através da pesquisa e encorajar a integração da informação biomédica com 0 conhecimento psicológico, fomentando e difundindo a área. Enfim, a Psicologia da Saúde, com base no modelo biopsicossocial, utiliza os conhecimentos das ciências biomédicas, da Psicologia Clínica e da Psicologia Social-comunitária (Remor, 1999 citado por Castro \& Bornholdt, 2004). Por isso, o trabalho com outros profissionais é imprescindível dentro dessa abordagem.

Essa área fundamenta seu trabalho principalmente na promoção e na educação para a saúde, que objetiva intervir com a população em sua vida cotidiana antes que haja riscos ou se instale algum problema de âmbito sanitário. Podemos enquadrar como sendo psicologia da saúde aquelas práticas que atuem numa integração da saúde mental com a saúde física e social do paciente. A psicologia da saúde talvez seja a própria consequiência lógica das transformações pelas quais passa a sociedade, a partir da construção de um modelo teórico de atuação inerente à realidade do paciente (Angerami-Camon \& cols., 2009, pp. 11-12; Castro \& Bornholdt, 2004).

Seja na esfera do serviço público ou nas instituições privadas, a Psicologia ligada à área da Saúde, na realidade brasileira, ainda tem uma história significativamente recente. Há pouco mais de trinta anos começaram a se alocar de forma sistemática e oficial os primeiros psicólogos em instituições públicas de saúde no Brasil (Castro \& Bornholdt, 2004; Santos \& Vilela, 2009). Muitas questões, por exemplo, permanecem sem esclarecimento, dificultando a atuação do psicólogo, o ensino da prática no hospital, a inserção desse profissional nas equipes de saúde, a realização de pesquisas e, o mais grave, a consideração real da especialidade (Angerami-Camom e cols., 2009, p. 74). Segundo Angerami-Camon e cols. (2009), o psicólogo da saúde deve inserir-se nas equipes de saúde, não em um movimento de simplesmente incluir-se, mas sim de fixar-se, afirmar-se, interagir.

Este estudo é uma proposta de investigação sobre como a prática da Psicologia é considerada em setores de saúde através de dois olhares: a percepção do psicólogo sobre a própria prática em 
equipes multidisciplinares e a observação das equipes multidisciplinares sobre a Psicologia.

\section{Método}

Participantes

Para este trabalho uma amostra de conveniência foi composta por 29 psicólogos e 36 profissionais das demais áreas da saúde, incluindo médicos, enfermeiros, técnicos em enfermagem, nutricionistas e assistentes sociais. As Tabelas 1 e 2 apresentam as características demográficas dos participantes.

Tabela 1

\begin{tabular}{l|c} 
Dados Sociodemográficos dos Psicólogos & Freqüência \\
\hline Sexo & 26 \\
Feminino & 3 \\
Masculino & \\
Idade & 14 \\
22 a 31 anos & 8 \\
32 a 41 anos & 7 \\
42 a 55 anos & \\
Tempo de atuação & 8 \\
Seis meses a dois anos & 4 \\
Três a cinco anos & 17 \\
Acima de cinco anos & \\
Instituição em que trabalha & 23 \\
Publica & 0 \\
Particular & 6 \\
Ambas & \\
Local de atuação & \\
Ambulatório & 3 \\
Enfermaria & 6 \\
UTI & 1 \\
Múltiplos & 19 \\
Formação & \\
Graduação & \\
Pós Graduação & \\
Especialização & \\
Outros & 5 \\
\hline
\end{tabular}




\section{Tabela 2}

Dados Sociodemográficos dos Psicólogos Freqüiência

Sexo
Feminino
Masculino
Idade
22 a 31 anos
32 a 41 anos
42 a 55 anos

2 a 55 anos

Tempo de atuação

Seis meses a dois anos

Três a cinco anos

Acima de cinco anos

Instituição em que trabalha

Publica

Particular

Local de atuação

Ambulatório

Enfermaria

Emergência

UTI

Múltiplos

Formação

Graduação

Pós Graduação

Especialização

Outros

Instrumentos

Foram utilizados dois questionários desenvolvidos especificamente para este estudo, um para psicólogos e outro para os profissionais da área da saúde, disponibilizados online ou respondidos presencialmente. Cada questionários foi desenvolvido pelas próprias pesquisadoras, a partir da vivência da atuação em saúde. O questionário para psicólogos foi composto por 31 itens (dez questões dissertativas e 21 questões objetivas, além de dados sociodemográficos). O questionário para profissionais da área da saúde foi constituído por 30 itens (dez questões dissertativas e 20 questões objetivas, além de questões sobre dados sociodemográficos). As questões objetivas foram estruturadas em escala Likert de cinco pontos: 1 indicava "discordo totalmente", 2 correspondia a "discordo", 3 indicava "não concordo nem discordo", 4 
correspondia a "concordo"e 5 indicava "concordo totalmente".

\section{Cuidados Éticos}

Para assegurar o cumprimento de normas éticas na condução de pesquisas com seres humanos, constantes na Resolução do Conselho Nacional de Saúde 196/96, o projeto de pesquisa foi submetido ao Comitê de Ética em Pesquisa (CEP), da Universidade Católica de Brasília (UCB), credenciado ao Comitê Nacional de Ética em Pesquisa (CONEP), do Conselho Nacional de Saúde. O projeto foi aprovado em 21 de novembro de 2011, sob o registro 229/2011. Os questionários permanecem em arquivo ao qual somente a pesquisadora tem acesso.

\section{Procedimento}

Os participantes foram convidados a responder aos questionários do presente estudo por meio de mensagens de emails, enviadas para grupos e profissionais com os quais os pesquisadores mantinham contato profissional. Foi solicitado aos participantes que encaminhassem o questionário online a outros colegas. Alguns questionários foram respondidos presencialmente, a partir de contatos pessoais com psicólogos e profissionais de saúde.

A primeira parte do questionário online apresentou o Termo de Consentimento Livre e Esclarecido. Caso o participante concordasse em participar, deveria assinalar sua concordância no campo especificado, pois somente após esta concordância o questionário seria apresentado no site. Participantes convidados pessoalmente para participar do estudo obtiveram inicialmente esclarecimentos sobre os objetivos da pesquisa, a partir da leitura do Termo de Consentimento Livre e Esclarecido. Caso concordassem em participar da pesquisa, poderiam assinar o Termo em duas vias e dar prosseguimento à aplicação do instrumento.

\section{Análise de dados}

Os dados obtidos a partir da aplicação dos questionários foram submetidos à análise estatística descritiva e inferencial, a partir do uso do software SPSS Statistical Package for the Social Sciences versão 16.0. 


\section{Resultados}

As comparações entre as médias de grupo de profissionais são apresentadas na

Tabela 3.

respostas do grupo de psicólogos e do

Tabela 3

Comparação entre respostas de profissionais psicólogos $(* p<0.05 ; * *<<0.01)$

\begin{tabular}{lcc}
\hline Questões & Profissionais & Psicólogos \\
& M(DP) & M(DP) \\
\hline Com quantos psicólogos trabalha & $0,55(0,87)^{* *}$ & $2,07(1,27)^{* *}$ \\
A psicologia é essencial na equipe multidisciplinar & $4,66(0,53)^{*}$ & $4,93(0,26)^{*}$ \\
Durante a formação acadêmica, é importante trabalhar com psicólogos & $4,58(0,60)^{*}$ & $4,89(0,31)^{*}$ \\
O psicólogo/outros profissionais contribuem para sua atuação & $4,35(0,77)$ & $4,58(0,68)$ \\
Você encontra dificuldades na comunicação com psicólogo/equipe & $2,71(1,02)^{* *}$ & $3,59(0,87)^{* *}$ \\
No seu trabalho existe comunicação multiprofissional & $3,95(0,88)^{*}$ & $3,35(1,07)^{*}$ \\
O modo de agir do psicólogo/seus colegas tem repercussão no seu agir & $3,78(1,00)$ & $4,13(0,69)$ \\
Deveria haver psicólogos em todos os setores das áreas da saúde & $4,53(0,74)$ & $4,55(0,63)$ \\
Você requisitaria um psicólogo/profissional para ajudar na atuação & $4,36(0,83)$ & $4,55(0,57)$ \\
A equipe funciona com mais eficiência com um psicólogo atuando & $4,11(0,94)$ & $4,13(0,69)$ \\
Existe diferença nos pacientes quando recebem atendimento psicológico & $4,51(0,65)$ & $4,68(0,47)$ \\
Você avalia que a intervenção do psicólogo junto à equipe ajuda na adesão & $4,51(0,66)$ & $4,69(0,47)$ \\
A sua prática tem repercussão no modo de agir de outros profissionais & $3,63(0,78)^{*}$ & $4,20(0,61)^{*}$ \\
Existe comunicação satisfatória na equipe & $3,61(1,02)^{* *}$ & $2,41(0,90)^{* *}$ \\
Você utiliza estratégias para se integrar aos demais profissionais & $3,23(1,07)^{* *}$ & $4,06(0,59)^{* *}$ \\
Sua formação foi orientada para o trabalho em equipe & $4,30(0,98)^{*}$ & $3,37(1,42)^{*}$ \\
\hline
\end{tabular}

Destacam-se algumas diferenças estatisticamente significativas entre os escores médios apresentados pelos participantes. Psicólogos indicaram trabalhar com mais profissionais de psicologia $(m=2,07)$ quando comparados a outros profissionais, que indicaram trabalhar em média com menos psicólogos na equipe $(m=0,55)$. Apesar de outros profissionais indicarem escassez de psicólogos na sua equipe foi atribuída grande importância ao trabalho deste em ambos os grupos, como pode ser observado nas médias elevadas verificadas nas questões: “A psicologia é essencial na equipe multidisciplinar"; e a questão "Durante a formação acadêmica, é importante trabalhar com psicólogos". 
Outro dado que merece destaque é a comunicação entre os membros da equipe, em que a média dos profissionais que indicaram dificuldades na comunicação com o psicólogo correspondeu a 2,71 enquanto para os psicólogos a média indicada foi 3,59. Sobre a repercussão da prática no modo de agir de outros profissionais, os psicólogos indicaram médias mais altas $(m=4,20)$, quando comparados aos outros profissionais $(m=3,63)$. Em relação à questão "existe comunicação satisfatória com a equipe", psicólogos indicaram médias mais baixas, $(m=2,41)$, quando comparados aos outros profissionais ( $m$ $=3,6)$.

Destaca-se ainda que em relação ao uso de estratégias para integrar-se aos demais profissionais, os psicólogos apresentaram uma média mais elevada $(m=4,06)$ que os outros profissionais $(m=3,23)$. Os psicólogos apresentaram média 3,37 quando questionados sobre sua formação orientada para o trabalho em equipe, enquanto outros profissionais apresentaram médias mais altas nesta questão $(m=4,30)$.

Características da amostra como idade, tempo de atuação e tipo de formação profissional indicaram possibilidade de comparações inferenciais entre os subgrupos, em cada variável. Em relação ao grupo de profissionais não psicólogos, não foram observadas diferenças estatisticamente significativas, à exceção das estratégias utilizadas para se integrar aos demais profissionais (questão 17). Participantes com menos tempo de atuação indicaram médias mais baixas neste quesito $(m=2,58, d p=1,08)$ quando comparados a profissionais com mais tempo de atuação $(m=3,66, d p=0,84)$ e a diferença entre médias foi estatisticamente significativa $(p=0,008)$.

Considerando o grupo de psicólogos, características como idade e tempo de atuação foram associadas de forma estatisticamente significativa a alguns resultados específicos do questionário. Para a questão relativa à existência de comunicação no seu local de trabalho, psicólogos com menos tempo de atuação indicaram médias mais baixas $(m=2,55, d p=0,79)$ do que psicólogos com mais tempo de atuação $(m=3,88, d p=$ 0,92 ) e tal diferença foi estatisticamente significativa $(p=0,001)$. Em relação à "existe diferença nos pacientes quando recebem atendimento do psicólogo", participantes com menos tempo de atuação indicaram médias mais elevadas $(m=4,91$, $d p=0,28)$ do que participantes com mais tempo de atuação $(m=4,52, d p=0,51)$ e 
esta diferença foi estatisticamente significativa $(p=0,026)$. Em relação à questão "existe comunicação satisfatória com a equipe", psicólogos com menos tempo de atuação indicaram médias mais baixas $(m=2,00, d p=0,73)$ do que psicólogos com mais tempo de atuação $(m=2,70, d p=0,91)$ e a diferença entre médias foi estatisticamente significativa $(p=0,036)$.

Considerando a idade dos psicólogos, participantes mais novos indicaram trabalhar com menos colegas psicólogos $(m=1,57 d p=1,39)$ quando comparados a participantes com mais idade $(m=2,53 d p=0,99)$ e a diferença entre escores foi estatisticamente significativa $(p=0,041)$. Considerando a importância de outros profissionais para a formação dos psicólogos, participantes mais novos indicaram pontuações mais elevadas $(m=4,85 d p=0,36)$ quando comparados a participantes com mais idade ( $m=4,33 d p=0,81)$ e esta diferença foi estatisticamente significativa $(p=0,036)$.

O questionário também investigou a percepção dos profissionais e dos psicólogos acerca do encaminhamento para o atendimento psicológico. Dezessete profissionais de outras áreas da saúde responderam que o encaminhamento feito por profissionais em geral, quatro pessoas responderam que são feitos por psicólogo e oito participantes responderam que são feitos de outras formas. Entre o grupo de psicólogos dois responderam que o encaminhamento é feito por profissionais em geral, dois indicaram que são feitos por psicólogos e 23 participantes responderam que é feito de outras formas.

Outra pergunta do questionário solicitava a opinião dos participantes acerca da implantação do sistema de atendimento psicológico a todos os pacientes. Trinta e três profissionais responderam que concordam com tal implantação, dois disseram ser indiferentes e nenhum não concorda. Vinte e três psicólogos afirmaram concordar com o atendimento a todos os pacientes, três se mostraram indiferentes e um não concorda com tal implantação. Ambos os grupos avaliam como positivo a implantação do atendimento psicológico.

A última questão do questionário retratou a percepção dos psicólogos a respeito de como os outros profissionais agem em relação ao seu trabalho. Onze psicólogos relataram que estão satisfeitos com o modo como os outros profissionais agem em relação ao seu trabalho, doze 
disseram ser indiferentes a isto e cinco não estão satisfeitos com o tratamento de outros profissionais em relação com seu trabalho. Sobre a percepção dos profissionais a respeito de como $o$ psicólogo age frente às situações que dele necessite, quinze profissionais estão satisfeitos, oito relataram ser indiferente e quatro não estão satisfeitos.

\section{Discussão}

A partir dos resultados da pesquisa encontram-se alguns índices sobre o cotidiano de trabalho das equipes multidisciplinares em contexto de atenção em saúde, em especial, a percepção de psicólogos e outros profissionais sobre contribuição da psicologia neste setor. Foram encontrados dados relevantes em relação à percepção dos outros profissionais de saúde em relação ao psicólogo e do psicólogo em relação à percepção dos outros profissionais sobre sua atuação.

A pesquisa revelou que há desejo das equipes de mais psicólogos atuando na atenção em saúde: entre os psicólogos, a pesquisa revelou que eles trabalham com um a dois psicólogos na equipe. Já entre os demais profissionais de saúde, essa média é menor. Essa realidade pode gerar a falta de percepção de outros profissionais sobre as contribuições da psicologia da saúde, corroborando os dados destacados por Dimenstein (1998), More e cols (2004) e também Santos e Vilela (2009) em relação à quantidade insatisfatória de psicólogos nas equipes de saúde.

Outro dado relevante na pesquisa está relacionado à estratégia de integração entre profissionais da equipe de saúde. A maioria dos psicólogos respondeu que emprega técnicas e procedimentos para integrar-se com os demais profissionais, tais como discussão de casos e reuniões em equipes. Entretanto, a média é menor entre os demais profissionais de saúde que consideram utilizar esses recursos para aumentar a integração. O resultado pode revelar que a realidade de trabalho multidisciplinar entre as equipes de saúde ainda se encontra distante de ser consolidado.

Uma das hipóteses está na formação durante a graduação dos diversos profissionais que atuam na saúde, inclusive os psicólogos. A falta de disciplinas em comum e trabalhos multidisciplinares ainda na graduação pode prejudicar o futuro a pensar no trabalho interdisciplinar. Saar e Trevizan (2007) atribuíram às instituições de ensino superior a responsabilidade pelo desconhecimento 
dos distintos papéis profissionais e pelo escasso entrosamento da equipe, uma vez que a academia não promove atividades conjuntas/integradas durante o processo de formação. Referente à comunicação entre as equipes, um dado curioso está relacionado à percepção sobre a compreensão de informações e comunicação entre os profissionais. Os psicólogos demonstraram sentir maior dificuldade em compreender os conteúdos trazidos pelos demais profissionais de saúde, comparado ao resultado gerado pelos demais profissionais, que parecem compreender melhor o discurso dos psicólogos. Outro dado semelhante está relacionado à satisfação com a comunicação entre equipes. Comparado aos outros profissionais de saúde, os psicólogos percebem que a comunicação entre equipe é mais insatisfatória frente aos demais profissionais de saúde, que acreditam que há melhor comunicação entre as equipes. Estes resultados destacam a necessidade de maior integração entre os profissionais nas equipes multidisciplinares, que possa ultrapassar barreiras de formação curricular e diferenças técnicas entre as áreas (Fossi \& Guareschi, 2004; More \& cols., 2004; Saar \& Trevizan, 2007; Santos \& Vilela, 2009).
Uma das hipóteses pode ser relacionada à formação do profissional em carreira (pertencimento, identidade, informações restrita, linguagem própria, e discurso restrito), os grupos sociais são formados geralmente por conhecimento em comum entre os membros dos grupos. Desta forma é natural que ocorra a exclusão de um indivíduo que não tem acesso ou não compreende as informações específicas de determinado grupo.

Os psicólogos acreditam que a psicologia é essencial na equipe multidisciplinar. Esses dizem que durante a formação acadêmica é importante trabalhar com profissionais de outras áreas da saúde e que a atuação de outros profissionais contribuem para sua própria atuação. Notase que os psicólogos percebem a valorização da sua atuação pelos demais profissionais.

Os dados encontrados nesta pesquisa se diferenciam dos resultados de outras pesquisas. Segundo Romano (1999 apud Tonetto \& Gomes, 2007) há queixas entre psicólogos de que muitas das suas observações clínicas não são prontamente aceitas pelas equipes. Tais dificuldades têm gerado discussões sobre qual o modo mais apropriado da Psicologia se inserir nas equipes multidisciplinares. Pode-se 
perceber que tal dificuldade não é encontrada com os profissionais que participaram desta pesquisa.

Observa-se que é de suma importância o psicólogo inserido nas equipes da saúde. Para os outros profissionais é de grande valor trabalhar com psicólogo durante a formação acadêmica e esses mesmos profissionais relatam que requisitaria o psicólogo para ajudar na sua atuação. Nessa mesma direção, Böing e Crepaldi (2010) apontam que os profissionais, à medida que se tornaram conscientes de que possuíam limites técnicos ou pessoais para enfrentar certos obstáculos, passaram a solicitar a ajuda da equipe de trabalho, o que transformou a resolução do problema e o enfrentamento da imprevisibilidade do processo saúde-doença em uma tarefa de equipe.

Muitos profissionais da saúde relatam sentir dificuldades em se relacionar com psicólogos, atribuídas principalmente à organização das equipes $\mathrm{e}$ dificuldades de interação comunicativa. No entanto, não se pode desprezar a tradição histórica do modelo biomédico e nem assumi-lo como obstáculo ao trabalho. A dinâmica da relação entre pacientes e profissionais da saúde fundamenta-se na herança de um modelo que atribui ao adoecer a influência apenas dos aspectos neurofisiológicos e bioquímicos do sistema nervoso, sem considerar a interferência dos fatores que envolvem as vivências históricas do paciente (Castro \& Bornholdt, 2004; De Marco, 2010; Feijó, 2006; More \& cols., 2004; Saar \& Trevizan, 2007; Santos \& Vilela, 2009).

Nota-se uma média alta em relação à possibilidade de haver psicólogos em todos os setores das áreas da saúde. Esses profissionais dizem que o atendimento do psicólogo faz diferença nos pacientes e que essa intervenção do psicólogo junto a equipe ajuda a adesão aos cuidados. Estes dados corroboram com os estudos de Angerami-Camom e cols. (2009), ao afirmar que cabe ao psicólogo a responsabilidade da conquista do contexto de saúde como campo de sua atuação profissional. E esta perspectiva apenas se tornará real quando sua reflexão o levar ao encontro de parâmetros que o conduzam a uma atuação permeada pela multidisciplinaridade institucional.

A pesquisa confirma que o trabalho em equipe é fundamental para o reconhecimento das diferentes áreas de atuação. Entretanto, a prática psicológica no contexto de saúde precisa ser ampliada, o que requer maior presença destes profissionais em diferentes equipes. Faz-se 
necessário que o psicólogo, participante das equipes de saúde mostre sua profissão através de seu pensar e agir, visando buscar maior contextualização das suas ações para que equipe tenha conhecimento coerente a respeito dessa ciência e profissão (Castro \& Bornholdt, 2004; De Marco, 2010; Feijó, 2006; Fossi \& Guareschi, 2004; Santos \& Vilela, 2009).
A comparação de resultados e dados obtidos indica a importância e a necessidade de novos estudos, de cunho interventivo, que possam modificar dinâmicas de interação em equipes multidisciplinares, a fim de aprofundar a percepção do psicólogo sobre sua atuação em saúde e a percepção dos demais profissionais sobre possíveis contribuições da Psicologia.

\section{Referências}

Angerami-Camom, V.A. (2009). Psicologia da Saúde: um novo significado para a prática clínica. (1 $1^{\mathrm{a}}$. ed). São Paulo: Cengage Learning.

BRASIL. Ministério da Saúde. Secretaria Nacional de Assistência Social à Saúde (1997). ABC do SUS: doutrinas e princípios. Brasília: Ministério da Saúde.

Böing, E. \& Crepaldi, M.A,. (2010). O Psicólogo na atenção básica: uma incursão pelas políticas públicas de saúde Brasileiras. Psicologia: Ciência e Profissão, 30(3), 634-649. DOI: $10.1590 / \mathrm{S} 1414-98932010000300014$.

Castro, E.K. \& Bornholdt, E. (2004). Psicologia da saúde x psicologia hospitalar: definiç̧̃̃es e possibilidades de inserção profissional. Psicologia: Ciência e Profissão, 24(3), 48-57. DOI: $10.1590 / \mathrm{s} 1414-98932004000300007$.

Czeresnia, D. (2003). O conceito de saúde e a diferença entre prevenção e promoção. Em: D. Czeresnia e C.M. Freitas (Orgs.), Promoção da Saúde: conceitos, reflexões, tendências (pp. 39-53). Rio de Janeiro: Editora Fiocruz.

Dimenstein, M.D.B. (1998). O psicólogo nas Unidades Básicas de Saúde: desafios para a formação e atuação profissionais. Estudos de Psicologia, 3(1), 53-81. DOI: 10.1590/S1413-294X1998000100004.

Feijó, G. (2006). A psicologia e o processo de humanização no hospital diante da relação enfermeiro- paciente. Trabalho de Conclusão de Curso de Graduação em Psicologia, 
Universidade do Sul de Santa Catarina, Palhoça.

Fossi, L.B. \& Guareschi, N.M.F. (2004). A psicologia hospitalar e as equipes multidisciplinares. Revista da SBPH, 7(1), 29-43.

De Marco, M.A. (2010). A face humana da medicina: do modelo biomédico ao modelo biopsicossocial. (2 ${ }^{\mathrm{a}}$. Ed). São Paulo: Casapsi.

More, C.L.O.O., Crepaldi, M.A., Queiroz, A.H., Wendt, N.C. \& Cardoso, V.S. (2004). As representações sociais do psicólogo entre os residentes do programa de saúde da família e a importância da interdisciplinaridade. Psicologia Hospitalar, 1(1), 59-75.

Rêgo, I.K.P. (2008). Psicoterapia no contexto hospitalar: uma proposta de atendimento psicológico para mulheres com miomatose uterina. Trabalho de Conclusão de Curso de Graduação em Psicologia, Universidade Estadual do Piauí, Teresina.

Romano, B.W. (1999). Princípios para a prática da psicologia clínica em hospitais. São Paulo: Casa do Psicólogo.

Saar, S.R.C. \& Trevizan, M.A. (2007). Papéis profissionais de uma equipe de saúde: visão de seus integrantes. Revista Latino-Americana de Enfermagem, 15(1), 1-7.

Santos, F.M.S. \& Jaco-Vilela, A.M. (2009). O psicólogo no hospital geral: estilos e coletivos de pensamento. Paideia, 19(43), 189-197. DOI: 10.1590/s0103-863x2009000200007.

Silva, L.B. (2008). Do violino à rabeca. Trabalho de Conclusão de Curso de Graduação em Psicologia, Universidade Do Vale Do Rio Dos Sinos, São Leopoldo.

Tonetto, A.M. \& Gomes, W.B. (2007). A prática do psicólogo hospitalar em equipe multidisciplinar. Estudos em Psicologia, 24(1), 89-98. DOI: 10.1590/s0103$166 \times 2007000100010$.

As autoras:

Marina Kohlsdorf é doutora e mestre em Processos de Desenvolvimento Humano e Saúde pelo Programa de Pós-Graduação em Processos de Desenvolvimento Humano e Saúde, no Instituto de Psicologia da Universidade de Brasília. Graduação em Psicologia pela Universidade de Brasília. Psicóloga na Unidade de Transplantes do Hospital Universitário de Brasília (HUB) de 2008 a 2012. Coordenadora adjunta do Curso de Especialização em Psicologia da Saúde (Instituto Brasiliense de Análise do Comportamento - IBAC) de 2010 a 2014. Docente de 2011 a 2013 no curso de Psicologia da Universidade Católica de Brasília - UCB. Professora no curso de Psicologia do Centro Universitário UniCeub nos cursos de graduação e mestrado em Psicologia e membro do Comitê de Análise de Projetos de Pesquisa na mesma instituição. Psicóloga na Secretaria de Saúde do Distrito Federal no Hospital Materno-Infantil de Brasília (HMIB). Colaboradora no Laboratório de Desenvolvimento em Condições Adversas - LADVERSA - na Universidade de Brasília (UnB). Editora associada da revista Psicologia: Reflexão e Crítica. E.mail: marinak@unb.br 


\section{MARINA KOHLSDORF, RAYANNE POLLYANE CHAGAS BORGES}

Rayanne Pollyane Chagas Borges é estudante de psicologia da Universidade Católica de Brasília, há dois anos participa da pesquisa O Pré-Natal Psicológico como Programa de Prevenção a Depressão Pós-Parto. Atualmente é estagiaria do HBDF, UTI. E.mail: rpcb@ gmail.com

Recebido em: 25/02/2017.

Aprovado em: 20/06/2017. 